per year) versus 25 pneumonias (rate: 0.039 events per patient per year) with fixed triple and 353 exacerbations (0.565) versus 18 pneumonias (0.029) with Fostair (figure 1A). In TRINITY study, the number of events was 485 exacerbations $(0.472)$ versus 30 pneumonias $(0.029)$ with fixed triple and 569 exacerbations (0.583) versus 20 pneumonias (0.020) with tiotropium (figure 1B). Overall, treatment with fixed triple therapy reduced exacerbations by 65 events compared to Fostair (adjusted rate ratio: 0.773, $\mathrm{p}=0.005$ ) and by 84 events compared to tiotropium $(0.801, \mathrm{p}=0.003)$. No fatal pneumonias occurred in TRILOGY while 5 pneumonias led to death in TRINITY (1 with fixed triple versus 4 with tiotropium). All pneumonias were classified as non-related to treatment.

Conclusions This analysis confirms that, in two independent populations of COPD patients treated with an ICS containing extrafine fixed triple combination, the number of incident pneumonia remains very small compared to that of moderate/ severe exacerbations. The benefit observed in reducing the absolute number of exacerbations outweighs the increase observed in absolute number of pneumonias, thus confirming the positive risk benefit balance of extrafine fixed triple in severe/very severe COPD patients.

Please refer to page A260 for declarations of interest in relation to abstract P273.

\section{P274 COMPARISON OF THE INITIATION OF COPD TREATMENT WITH LICENSED FDC ICS/LABA TREATMENTS IN TERMS OF DISEASE CONTROL AND COST EFFECTIVENESS}

${ }^{1} S$ Wan Yau Ming, ${ }^{2}$ J Haughney, ${ }^{3} \mathrm{D}$ Ryan, ${ }^{4}$ I Small, ${ }^{5} \mathrm{~F}$ Lavorini, ${ }^{6} \mathrm{~K}$ Gruffydd-Jones, ${ }^{7} \mathrm{~A}$ Papi ${ }^{8} \mathrm{D}$ Singh, ${ }^{8} \mathrm{D}$ Halpin, ${ }^{9} \mathrm{~J}$ Hurst, ${ }^{10} \mathrm{~S}$ Patel, ${ }^{10} \mathrm{M}$ Ochel, ${ }^{1} \mathrm{D}$ Price. ${ }^{1}$ Observational and Pragmatic Research Institute, Singapore; ${ }^{2}$ Academic Primary Care, University of Aberdeen, Aberdeen, UK; ${ }^{3}$ Optimum Patient Care, UK; ${ }^{4}$ Peterhead Health Centre, Aberdeen, UK; ${ }^{5}$ Department of Critical Care, University of Florence, Florence, Italy; ${ }^{6}$ University of Bath, Bath, UK; ${ }^{7}$ University of Ferrara, Ferrara, Italy; ${ }^{8}$ Royal Devon and Exeter University Hospital, UK; ${ }^{9}$ University College London, London, UK; ${ }^{10}$ Chiesi Ltd, Singapore

\subsection{6/thoraxjnl-2017-210983.416}

Several fixed-dose combination inhaled corticosteroid (FDC ICS/ LABA) inhalers are licensed for chronic obstructive pulmonary disease (COPD) in the UK. This study compares effectiveness of Fostair 100/6 (BDP/FOR) metered dose inhalers (MDI) against other licensed FDC ICS/LABAs, namely; Seretide Accuhaler 500 (FP/ SAL) dry powder inhaler (DPI) and Symbicort Turbohaler 200/6 and 400/24 DPI (BUD/FOR) in a patient group with recent exacerbations and poor lung function. A historical cohort study using data extracted from the Optimum Patient Care Research Database. Patients with diagnostic read codes for COPD, smoking history, age $\geq 35$ years, postdose $\mathrm{FEV}_{1}$ percent predicted $<55 \%, \geq 1$ previous long acting bronchodilator ever, and $\geq 1$ previous exacerbation in the 18 months prior initiation of FDC ICS/LABA therapy. The observation period consisted of one year after the initiation of FDC ICS/LABA for follow up and one year prior for patient characterisation. Patients were excluded if they switched or halted ICS/LABA treatment during the 1 year follow up period. The primary outcome was the proportion of patients with $\geq 1$ exacerbation. The non-inferiority margin was defined as $20 \%$. Patients were directly matched $1: 1$ on categorised age, smoking status, $\mathrm{FEV}_{1}$ percent predicted and exacerbations. Mean total costs consisting of medication/resource costs were compared between treatment arms after adjusting for confounders. Out of the patients prescribed BDP/FOR $(n=537)$ and 537 FP/SAL ( $\mathrm{n}=537)$, the median age was 70 and 69 respectively and $41.7 \%$ were current smokers. In the BDP/FOR $(n=540)$ and BUD/ FOR $(n=540)$ comparison, the median age was 70 and 69 respectively, and $42 \%$ were current smokers. The risk of $\geq 1$ exacerbation in BDP/FOR group was non-inferior during the year following the initiation of ICS/LABA compared to FP/ SAL (OR 0.89, 95\% CI: 0.67-1.19) or BUD/FOR (OR 0.79, 95\% CI: 0.58-1.08). Cost was significantly lower for BDP/FOR versus FP/SAL (adjusted mean $£ 730.0$ versus $£ 850.1$ respectively, $\mathrm{p}<0.001$ ) and lower for BDP/FOR versus BUD/FOR (adjusted mean $£ 732.4$ versus $£ 757.2$ respectively, $\mathrm{p}=0.054$ ). Treatment with $\mathrm{BDP} / \mathrm{FOR}$ is non-inferior in terms of exacerbation risk and is additionally associated with a lower point estimate of exacerbation risk, a lower cost compared to FP/SAL and BUD/FOR, and a lower ICS dose compared to FP/SAL.

\section{P275 COMPARING CLINICALLY RELEVANT IMPROVEMENT WITH UMECLIDINIUM/VILANTEROL AND TIOTROPIUM/ OLODATEROL IN SYMPTOMATIC COPD: A RANDOMISED NON-INFERIORITY CROSSOVER TRIAL}

${ }^{1} \mathrm{C}$ Compton, ${ }^{2} \mathrm{G}$ Feldman, ${ }^{3} \mathrm{AR}$ Sousa, ${ }^{4} \mathrm{D}$ Lipson, ${ }^{5}$ I Naya, ${ }^{6} \mathrm{~L}$ Tombs, ${ }^{1} \mathrm{~S}$ Patel, ${ }^{7} \mathrm{~B}$ Alcázar Navarrete. 'Global Respiratory Franchise, GSK, Brentford, UK; ${ }^{2}$ South Carolina Pharmaceutical Research, Spartanburg, US; ${ }^{3}$ Discovery Medicine, GSK, Uxbridge, UK; ${ }^{4}$ Respiratory Research and Development, GSK, King of Prussia, US; ${ }^{5}$ Respiratory Medicine, GSK, Brentford, UK; ${ }^{6}$ GSK, Uxbridge, UK; ${ }^{7}$ Neumología, Hospital de Alta Resolución de Loja, Granada, Spain

\subsection{6/thoraxjnl-2017-210983.417}

Introduction and Objectives Here we report the Results of the first direct comparison of the once-daily fixed-dose long-acting muscarinic antagonist/long-acting $\beta_{2}$-agonist (LAMA/LABA) combinations umeclidinium/vilanterol (UMEC/VI) 62.5/25 mcg and tiotropium/olodaterol (TIO/OLO) $5 / 5 \mathrm{mcg}$ in patients with chronic obstructive pulmonary disease (COPD).

Methods This randomised, 2-period crossover study (204990, NCT02799784) included inhaled corticosteroid-free patients with COPD, a modified Medical Research Council dyspnoea score $\geq 2$, forced expiratory volume in $1 \mathrm{~s}\left(\mathrm{FEV}_{1}\right) /$ forced vital capacity ratio of $<0.70$ and post-salbutamol $\mathrm{FEV}_{1} 50 \%-70 \%$ predicted. Patients were randomised to UMEC/VI (62.5/25 mcg once daily) via an ELLIPTA dry powder inhaler followed by TIO/OLO $5 / 5 \mathrm{mcg}$ (2 puffs once daily) via a RESPIMAT inhaler (each for 8 weeks with an interim 3 week washout period), or vice versa. The primary endpoint was change from baseline (CFB) in trough $\mathrm{FEV}_{1}$ at Week 8 with a non-inferiority (NI) margin of $-50 \mathrm{~mL}$ in the per protocol (PP) population. Additional outcomes included inspiratory capacity (IC), rescue medication use and ease of inhaler use (assessed using a six-point questionnaire). Adverse events (AEs) were also assessed.

Results 236 patients (mean age 64.4 years, 60\% male) were included in the intent-to-treat (ITT) population and 227 in the PP population. The primary endpoint of $\mathrm{CFB}$ in trough $\mathrm{FEV}_{1}$ at Week 8 confirmed NI of UMEC/VI vs TIO/OLO $(175 \mathrm{~mL}$ vs $122 \mathrm{~mL}$; least squares mean difference $53 \mathrm{~mL}$ [95\% confidence interval: 26, 80]; $\mathrm{p}<0.001$; PP population) and demonstrated superiority in the ITT population (Table). Patients receiving UMEC/VI were significantly more likely to achieve clinically meaningful improvements $(\geq 100 \mathrm{~mL})$ in trough $\mathrm{FEV}_{1}$ at Weeks 4 and 8 vs TIO/OLO, and showed significant improvements at Weeks 4 and 8 in IC and rescue medication use (Table). The 